\title{
A Decentralized Fault Detection and Prediction Scheme for Nonlinear Interconnected Continuous-time Systems
}

\author{
Hasan Ferdowsi, Deepthi L. Raja, and S. Jagannathan
}

\begin{abstract}
Complex nonlinear systems such as an aircraft, trains, automobiles, power plants and chemical plants are represented as nonlinear interconnected subsystems. Therefore, in this paper a novel decentralized fault diagnosis and prognosis (FDP) methodology is proposed for such large-scale systems. Current FDP approaches require the knowledge of the entire state or its estimated vector. But the main goal in this work is to design a local fault detector (LFD) or observer for each subsystem based on the measured local states of the subsystem alone. A local residual signal is generated via the measured states of the local subsystem and the estimated states provided by the LFD. A fault is detected when this local residual exceeds a predefined threshold. The adaptive online approximator in each LFD is activated upon detection to compensate the fault dynamics due to local and non-local faults. A novel update law for tuning the parameters of the online approximator is derived. Upon detection, faults local to the subsystem and to other subsystems are isolated. In addition, the proposed scheme provides the time to failure (or remaining useful life) information by using local measurements and the parameter update law of the LFD. Simulation results verify the effectiveness of the proposed decentralized FDP scheme.
\end{abstract}

\section{INTRODUCTION}

With current technological trends, practical systems that are of interest are large-scale and/or physically distributed, and are mostly referred to as large-scale interconnected systems. In these systems, the decomposition and spatial distribution of highly demanding computational tasks, one of which is the fault diagnosis, are of critical importance. In this paper, we present a decentralized fault diagnosis and prognosis scheme for these large scale interconnected continuous-time systems.

The two dominant fault diagnosis (FD) schemes include data driven and model-based. Data driven methods [1] need healthy and faulty data from the system which in turn requires large amounts of data transmission and computation. By contrast, in the model-based methods [2], the mathematical model of the system along with the sensor measurements are utilized to detect and diagnose faults. Researchers have worked on model-based FD schemes, using adaptive estimators [3-5], neural network (NN) based estimators [6,7], Fuzzy observers $[8,9]$, and so on.

Hasan Ferdowsi (e-mail: hfqk6@mst.edu), Deepthi Shreeya Lathamaheswari Raja (e-mail: dlxh2@mail.mst.edu), and S. Jagannathan (e-mail: sarangap@,mst.edu) are with the Department of Electrical \& Computer Engineering, Missouri University of Science and Technology (formerly University of Missouri-Rolla), Rolla, MO, 65401, USA. Research supported in part by NSF I/UCRC on Intelligent Maintenance Systems and Intelligent Systems Center.
Centralized fault diagnosis schemes are not preferred for practical large-scale interconnected systems such as power generation and distribution systems, telecommunication networks, water distribution networks, aircraft hydraulic systems, fluid power systems, since it requires sensing, processing and transmission of a large number of variables measured from the various parts of the system. In other words, centralized schemes can be very expensive and time-consuming for such large-scale interconnected systems. Therefore, decentralized control of interconnected systems by using local state measurements are presented in many articles [10-12] while decentralized fault diagnosis is being dealt only recently.

In the current decentralized fault diagnosis schemes [13-15], a large-scale continuous-time system is decomposed into a set of smaller subsystems which are interconnected by unknown nonlinear functions. However, it is assumed that the interconnection functions are known and the entire estimated system state vector is available [13-15] which is drawback.

By contrast, our objective in this paper is to design a network of local fault detectors (LFD) or observers for nonlinear continuous-time systems so that each LFD monitors a single subsystem by making use of only the local information or states. In addition, uncertainties caused by external disturbances, unmodeled dynamics, and interconnection effects, are taken into account in the LFD. The class of faults considered includes both abrupt and incipient faults. Only minimal communication between the LFDs is required upon detection for the sake of fault isolation. In addition, time to failure (TTF) determination is developed for the purpose of prognosis. Consequently, the proposed fault diagnosis scheme is different than [3, 13-15]. Here, the design of decentralized fault diagnosis and prognosis (FDP) scheme for incipient faults is considered.

Detection of a fault in any subsystem is initiated with the generation of a local residual signal by comparing the estimated local states from the LFD, which serves as a local observer, with that of the measured system states, and when this residual exceeds a predefined threshold, a fault is detected regardless of its location. Upon detection, an online approximator [7] in the LFD is activated. This approximator uses the local states and learns the fault function at each subsystem. Subsequently, the boundedness of detection residual and parameter estimation error is demonstrated under mild assumptions.

Upon detection, a fault isolation algorithm is utilized to determine whether or not the fault is local. Local faults affect local measurements quicker than the non-local faults. Therefore by comparing the time when the residual crosses 
the detection threshold at each subsystem, the location of the fault is identified.

Accurate approximation of fault function and determination of the fault location, allows a good estimation of TTF of the system by comparing the parameter estimates against the user defined parameter limits [16]. The TTF scheme ensures that the system will not be in operation beyond this limit as it is unsafe.

Thus the major contributions of this paper include the development of a decentralized fault diagnosis scheme wherein a LFD only uses local measurements in contrast with centralized fault detection schemes [3-9] or the current distributed fault diagnosis schemes [13-15]. Each LFD is designed to detect faults and then determine if the fault is local or nonlocal. Furthermore TTF estimation is performed upon fault location determination whereas such scheme is not available in most of the model-based methods [2-5].

This paper is organized as follows. Section II gives a problem formulation for large-scale interconnected continuous-time systems. Section III proposes the decentralized fault detection and isolation scheme, while Section IV describes the TTF determination, and finally Section V reports simulation results verifying the proposed scheme.

\section{SYSTEM DESCRIPTION}

Consider the general nonlinear continuous-time system

$$
\dot{x}(t)=\omega(x(t), u(t))+\eta(x(t), u(t))+\Pi\left(t-t_{0}\right) h\left(x(t), u_{f}(t)\right)
$$

where $u \in \mathbb{R}^{m}$ is the control input vector, $x \in \mathbb{R}^{n}$ is the system state vector, $\omega: \mathbb{R}^{n} \times \mathbb{R}^{m} \rightarrow \mathbb{R}^{n}$ represents the nonlinear system dynamics, $\eta: \mathbb{R}^{n} \times \mathbb{R}^{m} \rightarrow \mathbb{R}^{n}$ represents the system uncertainties, and $h: \mathbb{R}^{n} \times \mathbb{R}^{m} \rightarrow \mathbb{R}^{n}$ represents a vector of possible fault dynamics.

Now suppose that this system is comprised of $\mathrm{N}$ interconnected subsystems, and the $\mathrm{i}^{\text {th }}$ subsystem dynamics is defined by

$$
\begin{aligned}
\dot{x}_{i}(t) & =f_{i}\left(x_{i}(t), u_{i}(t)\right)+g_{i}\left(x_{i}(t), \bar{x}_{i}(t), u_{i}(t)\right) \\
& +\eta_{i}(x(t), u(t))+\Pi\left(t-t_{0}\right) h_{i}\left(x_{i}(t), u_{i}(t)\right)
\end{aligned}
$$

where $u_{i} \in \mathbb{R}^{m_{i}}$ is the local control input vector, $x_{i} \in \mathbb{R}^{n_{i}}$ is the local state vector, $\bar{x}_{i} \in \mathbb{R}^{\bar{n}_{i}}$ is the vector of interconnection states, $f_{i}: \mathbb{R}^{n_{i}} \times \mathbb{R}^{m_{i}} \rightarrow \mathbb{R}^{n_{i}}$ and $g_{i}: \mathbb{R}^{n_{i}} \times$ $\mathbb{R}^{\bar{n}_{i}} \times \mathbb{R}^{m_{i}} \rightarrow \mathbb{R}^{n_{i}}$ represent the known local and unknown interconnection functions respectively, $\eta_{i}: \mathbb{R}^{n} \times \mathbb{R}^{m} \rightarrow \mathbb{R}^{n_{i}}$ denotes the system uncertainties, and $h_{i}: \mathbb{R}^{n_{i}} \times \mathbb{R}^{m_{i}} \rightarrow \mathbb{R}^{n_{i}}$ is the local fault function.

The time profile $\Pi\left(t-t_{0}\right)$ is modeled by

$$
\begin{aligned}
& \Pi\left(t-t_{0}\right)=\operatorname{diag}\left\{\Omega_{i_{1}}\left(t-t_{0}\right), \Omega_{i_{2}}\left(t-t_{0}\right), \ldots, \Omega_{i_{n}}\left(t-t_{0}\right)\right\} \text { where } \\
& \Omega_{i_{j}}\left(t-t_{0}\right)=\left\{\begin{array}{ll}
0, & \text { if } \tau<0 \\
1-e^{-\bar{K}_{j} \tau}, & \text { if } \tau \geq 0
\end{array} \text { for } j=1, \ldots, n_{i}\right.
\end{aligned}
$$

and $\bar{\kappa}_{j}$ is an unknown constant that represents the rate at which a fault occurs. A larger value of $\bar{\kappa}_{j}$ indicates that it is an abrupt fault. The use of such time profiles is common in fault diagnosis literature $[16,17]$. Next standard assumptions are needed in order to proceed.

Assumption 1: The modeling uncertainty is bounded, i.e. $\left\|\eta_{i}(x(t), u(t))\right\| \leq \eta_{i_{M}}, \forall(x, u) \in(\chi \times U), i=1,2, \ldots, N$, where $\eta_{i_{M}}$ is a positive known constant.

Remark 1: Assumption 1 is needed to define the detection threshold analytically and prevent false alarms.

Assumption 2: Fault functions can be expressed as linear in the unknown parameters (LIP) [18]. Then, the fault functions can be approximated by using two-layer NN with bounded activation functions and target weight parameters.

Assumption 3: In the healthy operating condition of the system, the interconnection terms are unknown but bounded above, i.e. $\left\|g_{i}\left(x(k), u_{i}(k)\right)\right\| \leq \bar{g}_{i_{M}}, \forall(x, u) \in(\chi \times U), i=$ $1,2, \ldots, N$, where $\bar{g}_{i_{M}}$ is a positive constant.

Remark 2: Assumption 3 is needed only for the derivation of detection threshold. After the occurrence of a fault, the interconnection term is no longer bounded by a constant, but it will be shown that it is bounded by a function of residual and parameter estimation errors as defined later.

Next the proposed fault detection and isolation scheme is introduced.

\section{FAULT DETECTION AND ISOLATION SCHEME}

\section{A. Fault detection:}

Now consider the nonlinear LFD or observer for the subsystem ' $i$ ' described by

$\dot{\hat{x}}_{i}(t)=-\lambda \hat{x}_{i}(t)+f_{i}\left(x_{i}(t), u_{i}(t)\right)+\hat{h}_{i}\left(x_{i}(t), u_{i}(t) ; \hat{\theta}_{i}(t)\right)+\lambda x_{i}(t)(2)$

where $\hat{x}_{i}(k) \in \mathbb{R}^{n_{i}}$ is the estimated local state vector, $\lambda$ is a positive constant, and $\hat{h}_{i}: \mathbb{R}^{n_{i}} \times \mathbb{R}^{p_{i} \times n_{i}} \rightarrow \mathbb{R}^{n_{i}}$ is the output of the local online approximator (OLA) with $\hat{\theta}_{i} \in \mathbb{R}^{p_{i} \times n_{i}}$ being its set of unknown parameters. Initial values of the LFD are taken as $\hat{x}_{i}(0)=\hat{x}_{i_{0}}, \hat{\theta}_{i}(0)=\hat{\theta}_{i_{0}}$, such that $\hat{h}_{i}\left(x_{i}, u_{i}, \hat{\theta}_{i_{0}}\right)=0, \forall x_{i} \in X_{i}, u_{i} \in U_{i}$.

Define the local detection residual, $e_{i}=x_{i}-\hat{x}_{i}$. Under healthy operating conditions, the local residual dynamics are described by

$$
\dot{e}_{i}(t)=-\lambda e_{i}(t)+g_{i}\left(x_{i}(t), \bar{x}_{i}(t), u_{i}(t)\right)+\eta_{i}(x(t), u(t))
$$

Since $g_{i}\left(x_{i}(t), \bar{x}_{i}(t), u_{i}(t)\right)$ and $\eta_{i}(x(t), u(t))$ are both bounded in the healthy operating condition, and by appropriate selection of $\lambda$, the local detection residual will remain bounded prior to occurrence of a fault.

In this work, neural networks (NNs) are used as online approximators. The online approximator for each subsystem is off prior to the detection of a fault. Upon detection, the output of each OLA in the LFD is described by $\hat{h}_{i}(t)=\hat{\theta}_{i}^{T}(t) \phi_{i}\left(x_{i}(t), u_{i}(t)\right)$, where $\phi_{i}(t)=\phi_{i}\left(x_{i}(t), u_{i}(t)\right)$ is a basis function such as sigmoid or RBF.

When the local detection residual exceeds the local detection threshold, $\rho_{i}$, a fault is declared active through the index operator $H(x)$ which is defined by 


$$
H(x)= \begin{cases}1 & \text { if } x \geq 0 \\ 0 & \text { if } x<0\end{cases}
$$

Then the OLA in a LFD that generates, $\hat{h}_{i}(k)$, is initiated and tuned online using the following update law

$$
\dot{\hat{\theta}}_{i}(t)=H\left[\left\|e_{i}(t)\right\|-\rho_{i}\right]\left(\alpha_{i} \phi_{i}(t) e_{i}^{T}(t)-\gamma_{i} \hat{\theta}_{i}(t)\right)
$$

Upon detection, the local detection residual dynamics becomes

$$
\begin{aligned}
\dot{e}_{i}(t)= & -\lambda e_{i}(t)+g_{i}\left(x_{i}(t), \bar{x}_{i}(t), u_{i}(t)\right)+\eta_{i}(x(t), u(t)) \\
& +h_{i}\left(x_{i}(t), u_{i}(t)\right)-\hat{h}_{i}\left(x_{i}(t), u_{i}(t) ; \hat{\theta}_{i}(t)\right)
\end{aligned}
$$

Asserting the LIP assumption on the fault function, the above equation can be rewritten as

$$
\begin{aligned}
\dot{e}_{i}(t) & =-\lambda e_{i}(t)+g_{i}\left(x_{i}(t), \bar{x}_{i}(t), u_{i}(t)\right)+\eta_{i}(x(t), u(t)) \\
& +\theta_{i}^{T}(t) \phi_{i}\left(x_{i}(t), u_{i}(t)\right)+\varepsilon_{i}(t)-\hat{\theta}_{i}^{T}(t) \phi_{i}\left(x_{i}(t), u_{i}(t)\right) \\
& =-\lambda e_{i}(t)+g_{i}\left(x_{i}(t), \bar{x}_{i}(t), u_{i}(t)\right)+\eta_{i}(x(t), u(t)) \\
& +\tilde{\theta}_{i}^{T}(t) \phi_{i}\left(x_{i}(t), u_{i}(t)\right)+\varepsilon_{i}(t)
\end{aligned}
$$

where $\tilde{\theta}_{i}(k)=\theta_{i}-\hat{\theta}_{i}(k)$ is the parameter estimation error and $\varepsilon_{i}(k)$ is the OLA approximation error.

Next the following lemma introduces an upper bound for the summation of OLA approximation errors, modeling uncertainties, and interconnection term. This lemma is needed later in the stability proof.

Lemma 1: The terms comprising of the OLA approximation error, $\varepsilon_{i}(t)$, modeling uncertainties, $\eta_{i}(x(t), u(t))$, and interconnection function $g_{i}\left(x_{i}, \bar{x}_{i}, u_{i}\right)$ are expressed according to

$$
\begin{aligned}
\| g_{i}\left(x_{i}(t), \bar{x}_{i}(t), u_{i}(t)\right) & +\varepsilon_{i}(t)+\eta_{i}(x(t), u(t)) \| \\
& \leq\left\|e_{i}(t)\right\|+\left\|e_{i}(0)\right\|+\frac{\phi_{i_{\max }}}{\lambda}\left\|\tilde{\theta}_{i}(t)\right\|
\end{aligned}
$$

Proof: Consider the residual dynamics

$$
\begin{aligned}
\dot{e}_{i}(t)= & -\lambda e_{i}(t)+g_{i}\left(x_{i}(t), \bar{x}_{i}(t), u_{i}(t)\right)+\eta_{i}(x(t), u(t)) \\
& +\tilde{\theta}_{i}^{T}(t) \phi_{i}\left(x_{i}(t), u_{i}(t)\right)+\varepsilon_{i}(t)
\end{aligned}
$$

where $\tilde{\theta}_{i}(t)=\theta_{i}-\hat{\theta}_{i}(t)$ represents the parameter estimation error. Solving this set of state equations we get

$$
\begin{aligned}
e_{i}(t) & =e^{-\lambda t} e_{i}(0)+\int_{0}^{t} e^{-\lambda(t-\tau)}\left(g_{i}\left(x_{i}(\tau), \bar{x}_{i}(\tau), u_{i}(\tau)\right)\right. \\
& \left.+\tilde{\theta}_{i}^{T}(\tau) \phi_{i}\left(x_{i}(\tau), u_{i}(\tau)\right)+\varepsilon_{i}(\tau)+\eta_{i}(x(\tau), u(\tau))\right) d \tau
\end{aligned}
$$

This equation can be rewritten as

$$
\begin{array}{r}
\int_{0}^{t} e^{-\lambda(t-\tau)}\left(g_{i}\left(x_{i}(\tau), \bar{x}_{i}(\tau), u_{i}(\tau)\right)+\varepsilon_{i}(\tau)+\eta_{i}(x(\tau), u(\tau))\right) d \tau \\
=e_{i}(t)-e^{-\lambda t} e_{i}(0)-\int_{0}^{t} e^{-\lambda(t-\tau)} \tilde{\theta}_{i}^{T}(\tau) \phi_{i}\left(x_{i}(\tau), u_{i}(\tau)\right) d \tau
\end{array}
$$

Since $\int_{0}^{t} e^{-\lambda(t-\tau)} d \tau=\frac{1-e^{-\lambda t}}{\lambda}$, and $t>0, \lambda>0$, we have $\int_{0}^{t} e^{-\lambda(t-\tau)} d \tau \leq \frac{1}{\lambda}$. By using this term and applying the Frobenius norm we get

$$
\begin{aligned}
\| g_{i}\left(x_{i}(t), \bar{x}_{i}(t), u_{i}(t)\right) & +\varepsilon_{i}(t)+\eta_{i}(x(t), u(t)) \| \\
& \leq\left\|e_{i}(t)\right\|+\left\|e_{i}(0)\right\|+\frac{\phi_{i_{\max }}}{\lambda}\left\|\tilde{\theta}_{i}(t)\right\|
\end{aligned}
$$

The stability of the proposed observer is stated in the next theorem.

Theorem 1 (Local Fault Detection Observer Performance): Let the proposed local FD observer defined in (2) be used to monitor the subsystem described by (1), with the local OLA being turned on upon the detection of the fault. Let the update law in (3) be used to update the unknown parameter vector $\hat{\theta}_{i}(t$. In the presence of system uncertainties, the local FD residual, $e_{i}(t)$, and the parameter estimation error, $\tilde{\theta}_{i}(t)$, will be uniformly ultimately bounded, provided the design parameters are selected as

$$
\begin{gathered}
\lambda^{2}-\frac{3}{2} \lambda-\frac{\phi_{i_{\max }}}{2}>0 \\
\lambda>\frac{\alpha_{i} \phi_{i_{\max }}}{\gamma_{i}}
\end{gathered}
$$

Proof: Consider the following Lyapunov function candidate

$$
V(t)=\frac{1}{2} e_{i}^{T}(t) e_{i}(t)+\frac{1}{2 \alpha_{i}} \operatorname{tr}\left\{\tilde{\theta}_{i}^{T}(t) \tilde{\theta}_{i}(t)\right\}
$$

The first derivative of the Lyapunov function is given by

$$
\dot{\mathrm{V}}_{i}(t)=e_{i}^{T}(t) \dot{e}_{i}(t)+\frac{1}{\alpha_{i}} \operatorname{tr}\left\{\tilde{\theta}_{i}^{T}(t) \tilde{\theta}_{i}(t)\right\}
$$

Substitute $\dot{e}_{i}(t)$ from the local residual dynamics and noting $\dot{\tilde{\theta}}_{i}(t)=-\dot{\hat{\theta}}_{i}(t)$ from the parameter update law, in $\dot{\mathrm{V}}_{i}$ to arrive at

$$
\begin{gathered}
\begin{array}{r}
\dot{\mathrm{V}}_{i}(t)=e_{i}^{T}(t)\left(-\lambda e_{i}(t)+g_{i}\left(x_{i}(t), \bar{x}_{i}(t), u_{i}(t)\right)+\varepsilon_{i}(t)\right. \\
\left.+\eta_{i}(x(t), u(t))+\tilde{\theta}_{i}^{T}(t) \phi_{i}\left(x_{i}(t), u_{i}(t)\right)\right)
\end{array} \\
+\frac{1}{\alpha_{i}} \operatorname{tr}\left\{\tilde{\theta}_{i}^{T}(t)\left(-\alpha_{i} \phi_{i}(t) e_{i}^{T}(t)+\gamma_{i} \hat{\theta}_{i}(t)\right)\right\} \\
=-\lambda e_{i}^{T}(t) e_{i}(t)+\frac{1}{\alpha_{i}} \operatorname{tr}\left\{\tilde{\theta}_{i}^{T}(t)\left(\gamma_{i}\left(\theta_{i}-\tilde{\theta}_{i}(t)\right)\right)\right\} \\
+e_{i}^{T}(t)\left(g_{i}\left(x_{i}(t), \bar{x}_{i}(t), u_{i}(t)\right)+\eta_{i}(x(t), u(t))+\varepsilon_{i}(t)\right) \\
+e_{i}^{T}(t) \tilde{\theta}_{i}^{T}(t) \phi_{i}\left(x_{i}(t), u_{i}(t)\right)-e_{i}^{T}(t) \tilde{\theta}_{i}^{T}(t) \phi_{i}\left(x_{i}(t), u_{i}(t)\right)
\end{gathered}
$$

Therefore

$$
\begin{aligned}
\dot{\mathrm{V}}_{i}(t) & =-\lambda\left\|e_{i}(t)\right\|^{2}-\frac{\gamma_{i}}{\alpha_{i}}\left\|\tilde{\theta}_{i}(t)\right\|^{2} \\
& +e_{i}^{T}(t)\left(g_{i}\left(x_{i}(t), \bar{x}_{i}(t), u_{i}(t)\right)+\eta_{i}(x(t), u(t))+\varepsilon_{i}(t)\right) \\
& +\frac{\gamma_{i}}{\alpha_{i}} \operatorname{tr}\left\{\tilde{\theta}_{i}^{T}(t) \theta_{i}\right\}
\end{aligned}
$$


Taking the Frobenius norm and using Lemma 1, to arrive at $\dot{\mathrm{V}}_{i}(t) \leq-\lambda\left\|e_{i}(t)\right\|^{2}-\frac{\gamma_{i}}{\alpha_{i}}\left\|\tilde{\theta}_{i}(t)\right\|^{2}+\left\|e_{i}(t)\right\|\left(\left\|e_{i}(t)\right\|+\left\|e_{i}(0)\right\|+\frac{\phi_{i_{\text {max }}}}{\lambda}\left\|\tilde{\theta}_{i}(t)\right\|\right)$ $+\frac{\gamma_{i}}{\alpha_{i}}\left\|\tilde{\theta}_{i}(t)\right\|\left\|\theta_{i}\right\|$

Now we use the inequality $\|a\|\|b\| \leq \frac{1}{2}\|a\|^{2}+\frac{1}{2}\|b\|^{2}$

$$
\begin{aligned}
\dot{\mathrm{V}}_{i}(t) \leq & -\lambda\left\|e_{i}(t)\right\|^{2}+\left\|e_{i}(t)\right\|^{2}+\frac{1}{2}\left\|e_{i}(t)\right\|^{2} \\
& +\frac{1}{2}\left\|e_{i}(0)\right\|^{2}+\frac{\phi_{i_{\max }}}{2 \lambda}\left\|e_{i}(t)\right\|^{2}+\frac{\phi_{i_{\max }}}{2 \lambda}\left\|\tilde{\theta}_{i}(t)\right\|^{2} \\
& -\frac{\gamma_{i}}{\alpha_{i}}\left\|\tilde{\theta}_{i}(t)\right\|^{2}+\frac{\gamma_{i}}{2 \alpha_{i}}\left\|\tilde{\theta}_{i}(t)\right\|^{2}+\frac{\gamma_{i}}{2 \alpha_{i}}\left\|\theta_{i}\right\|^{2}
\end{aligned}
$$

After combining similar terms we get

$$
\begin{aligned}
\dot{\mathrm{V}}_{i}(t) \leq & -\left(\lambda-\frac{3}{2}-\frac{\phi_{i_{\max }}}{2 \lambda}\right)\left\|e_{i}(t)\right\|^{2}-\left(\frac{\gamma_{i}}{2 \alpha_{i}}-\frac{\phi_{i_{\max }}}{2 \lambda}\right)\left\|\tilde{\theta}_{i}(t)\right\|^{2} \\
& +\frac{\gamma_{i}}{2 \alpha_{i}}\left\|\theta_{i}\right\|^{2}+\frac{1}{2}\left\|e_{i}(0)\right\|^{2}
\end{aligned}
$$

Therefore, $\dot{\mathrm{V}}_{i}(t) \leq 0$, if one of the following conditions are also satisfied

$$
\left\|e_{i}(t)\right\|^{2}>\frac{D_{M}}{\lambda-\frac{3}{2}-\frac{\phi_{i_{\max }}}{2 \lambda}} \quad \text { or } \quad\left\|\tilde{\theta}_{i}(t)\right\|^{2}>\frac{D_{M}}{\frac{\gamma_{i}}{2 \alpha_{i}}-\frac{\phi_{i_{\max }}}{2 \lambda}}
$$

where $D_{M}=\frac{\gamma_{i}}{2 \alpha_{i}}\left\|\theta_{i}\right\|^{2}+\frac{1}{2}\left\|e_{i}(0)\right\|^{2}$ provided (4) and

holds. Hence, the local FD residual, $e_{i}(t)$, and the parameter estimation error, $\tilde{\theta}_{i}(t)$, are uniformly ultimately bounded.

\section{B. Fault isolation}

Theorem 1 guaranties the stability of the local FD residual and parameter estimation errors. When a fault is detected by a decentralized FD scheme in a complex large-scale system, its location need to identified since a fault in a particular subsystem can affect other subsystems through the interconnection terms causing the decentralized detection scheme to detect faults in several subsystems. Thus, the location of the fault would help isolate other subsystems from being affected by the fault.

The idea of our fault isolation scheme is based on the fact that there is a stronger correlation between the local fault magnitude and corresponding output magnitude. Local faults affect local measurements quicker than non-local faults as they have shorter propagation delay. Consequently, the fault location can be determined upon how quickly a fault is detected by the LFD. Each LFD will provide a different detection time based on its own perception of when the fault has occurred. In this method, each LFD will send its detection time to a central decision making unit. This unit will find the subsystem where the fault has been detected earliest by comparing all the detection times.

The process of fault isolation is illustrated by the flowchart shown in Fig. 1. In this flowchart, only two of the subsystems and their corresponding local fault detectors are shown, due to the limited space, but the general scheme involves all the subsystems. The isolation of faults with this method requires minimal communication between subsystems instead of transmitting the entire system state or its estimated values of all subsystems.

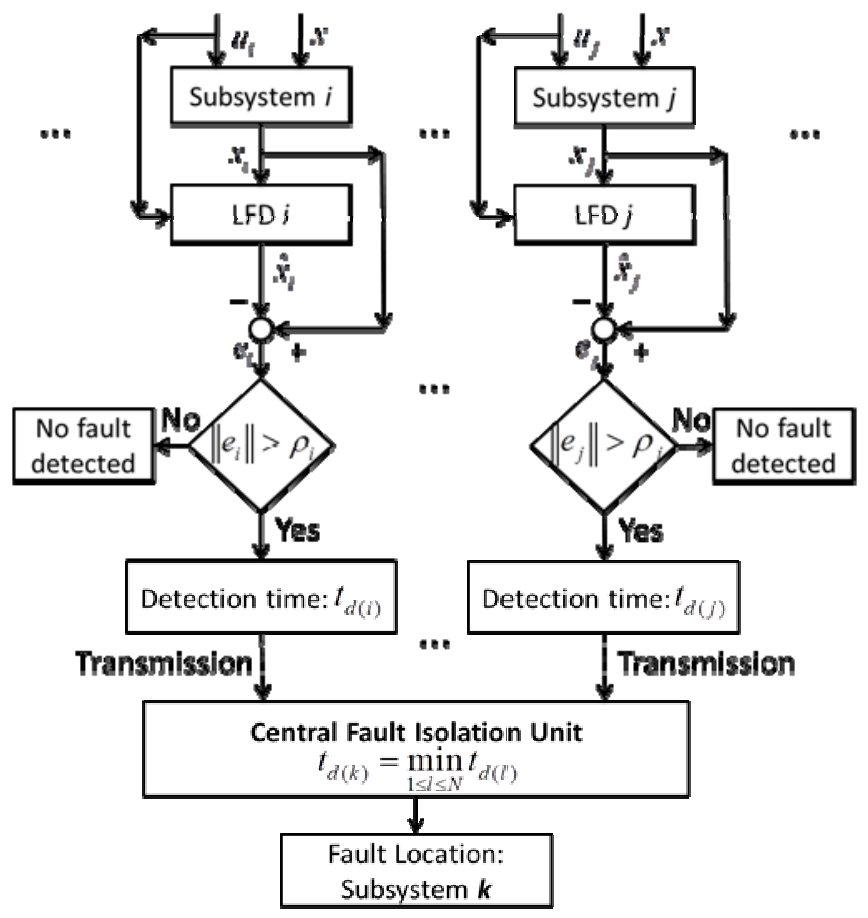

Fig. 1: Flow chart of fault isolation scheme

\section{PREDICTION SCHEME}

In the previous section, it was shown that the proposed LFD or fault detection observer can estimate the system states while the online approximator is able to estimate the local fault function upon detection of a fault. The next important step is to perform a prediction on time-to-failure, i.e, the time interval after which the system reaches a point of failure. After determination of fault location, we can use the parameter update law to find an estimation of time-to-failure.

The unknown parameters of the fault represent physical parameters. Each of these parameters could have a failure limit. In the next theorem, it is shown that by comparing the estimated parameter values at each time instant, with their failure limits and utilizing extrapolation, time-to-failure can be determined analytically.

Theorem 2 (TTF Determination): If the fault location is found to be the $i^{\text {th }}$ subsystem, TTF for the $j^{\text {th }}$ parameter of the $k^{\text {th }}$ fault, at the time $t$, can be determined by

$$
T T F_{j, k}(t)=\frac{1}{\gamma_{i}} \ln \left(\frac{\gamma_{i} \hat{\theta}_{i_{j, \mathrm{k}}}(t)-\alpha_{i} \phi_{i_{j}}(t) e_{i_{k}}^{T}(t)}{\gamma_{i} \theta_{i_{\mathrm{j}, \mathrm{k} \max }}-\alpha_{i} \phi_{i_{j}}(t) e_{i_{k}}^{T}(t)}\right)
$$

where $\theta_{i_{\mathrm{j}, \mathrm{k} \text { max }}}$ is the failure limit regarding the $j^{\text {th }}$ parameter of the $k^{\text {th }}$ fault in the $i^{\text {th }}$ subsystem, in terms of maximum value of the system parameter, $\theta_{i_{\mathrm{j}, \mathrm{k}}}$.

Proof: Upon detection, consider the parameter update law

$$
\dot{\hat{\theta}}_{i}(t)=-\gamma_{i} \hat{\theta}_{i}(t)+\alpha_{i} \phi_{i}(t) e_{i}^{T}(t)
$$


Since $\gamma_{i}>0$, this is clearly the state space representation of a stable system. Therefore $\hat{\theta}_{i}(t)$ will be bounded. The solution to the parameter update law is given by

$$
\hat{\theta}_{i}(t)=e^{-\gamma_{i}\left(t-t_{0}\right)} \hat{\theta}_{i}\left(t_{0}\right)+\int_{t_{0}}^{t} e^{-\gamma_{i}(t-\tau)} \alpha_{i} \phi_{i}(\tau) e_{i}^{T}(\tau) d \tau
$$

It was demonstrated in Theorem 1 that the local detection residual is bounded. We also know that the basis function has an upper limit. Therefore $\alpha_{i} \phi_{i}(\tau) e_{i}^{T}(\tau)$ is bounded, so we can hold it constant at time $t$ and describe $\hat{\theta}_{i}\left(t_{f}\right)$ by

$$
\begin{aligned}
\hat{\theta}_{i}\left(t_{f}\right) & =e^{-\gamma_{i}\left(t_{f}-t\right)} \hat{\theta}_{i}(t)+\alpha_{i} \phi_{i}(t) e_{i}^{T}(t) \int_{t}^{t_{f}} e^{-\gamma_{i}\left(t_{f}-\tau\right)} d \tau \\
& =e^{-\gamma_{i}\left(t_{f}-t\right)} \hat{\theta}_{i}(t)+\alpha_{i} \frac{1-e^{-\gamma_{i}\left(t_{f}-t\right)}}{\gamma_{i}} \phi_{i}(t) e_{i}^{T}(t)
\end{aligned}
$$

Therefore, $\hat{\theta}_{i_{j, \mathrm{k}}}\left(t_{f}\right)$ can be represented by

$\hat{\theta}_{i_{j, \mathrm{k}}}\left(t_{f}\right)=\frac{\alpha_{i}}{\gamma_{i}} \phi_{i_{j}}(t) e_{i_{k}}^{T}(t)+e^{-\gamma_{i}\left(t_{f}-t\right)}\left(\hat{\theta}_{i_{j, \mathrm{k}}}(t)-\frac{\alpha_{i}}{\gamma_{i}} \phi_{i_{j}}(t) e_{i_{k}}^{T}(t)\right)$ where $e_{i_{k}}$ is the $k^{\text {th }}$ element of the $i^{\text {th }}$ subsystem detection residual and $\phi_{i_{j}}$ is the $j^{\text {th }}$ element of the $i^{\text {th }}$ subsystem fault basis function. After substituting $\hat{\theta}_{i_{j, \mathrm{k}}}\left(t_{f}\right)$ with $\theta_{i_{\mathrm{j}, \mathrm{k} \max }}$ we $\operatorname{get} \frac{\gamma_{i} \theta_{i_{\mathrm{j}, \mathrm{kmax}}}-\alpha_{i} \phi_{i_{j}}(t) e_{i_{k}}^{T}(t)}{\gamma_{i} \hat{\theta}_{i_{j, \mathrm{k}}}(t)-\alpha_{i} \phi_{i_{j}}(t) e_{i_{k}}^{T}(t)}=e^{-\gamma_{i}\left(t_{f}-t\right)}$

With simple mathematical manipulations we arrive at

$$
T T F_{j, k}(t)=\frac{1}{\gamma_{i}} \ln \left(\left|\frac{\gamma_{i} \hat{\theta}_{i_{j, \mathrm{k}}}(t)-\alpha_{i} \phi_{i_{j}}(t) e_{i_{k}}^{T}(t)}{\gamma_{i} \theta_{i_{\mathrm{j}, \mathrm{k} \max }}-\alpha_{i} \phi_{i_{j}}(t) e_{i_{k}}^{T}(t)}\right|\right)
$$

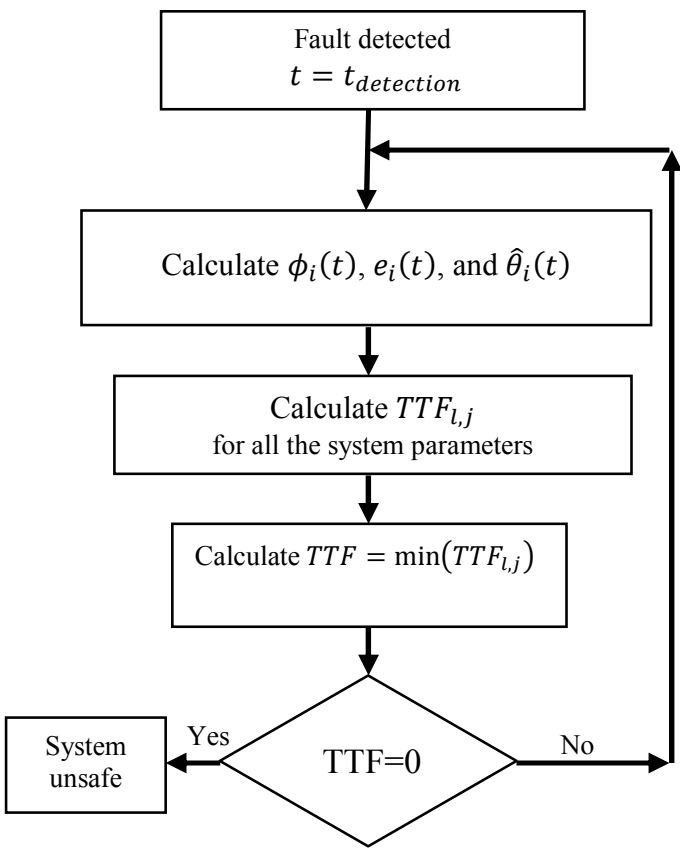

Fig. 2: Flow chart of the TTF determination.
Fig. 2 illustrates the process of finding the TTF after a local fault is detected. At each time instant, the local residual, fault basis function (which is the same basis function used in the parameter update law), and parameter estimates are found, and then time-to-failure for each of the parameters is calculated using the proposed formula for TTF determination. Then the overall time-to-failure is found by taking the minimum among time-to-failures for all the parameters. This is because the entire system will fail even if only one of the parameters reaches its failure limit.

\section{Simulation Results}

In this section, a five-tank water system [3] which is shown in Fig. 3, is used to verify the proposed decentralized fault detection and prediction scheme in simulations. This system consists of five connected tanks and two water inputs. It can be decomposed into two overlapping subsystems; subsystem 1 includes tanks 1, 2, and 3. And subsystem 2 includes tanks 3,4 , and 5 .

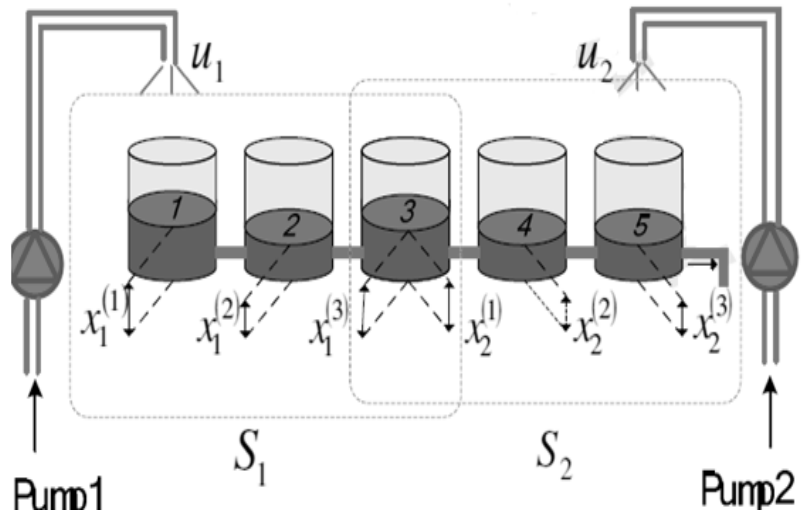

Fig. 3: Five tank benchmarking system.

The system dynamics are described by [3]

$$
\begin{aligned}
& \dot{x}_{1}^{(1)}(t)=\frac{1}{A}\left(u_{1}-c \operatorname{sis} \operatorname{sign}\left(x_{1}^{(1)}(t)-x_{1}^{(2)}(t)\right) \sqrt{2 g\left|x_{1}^{(1)}(t)-x_{1}^{(2)}(t)\right|}\right)+\eta_{1}(t) \\
& \dot{x}_{1}^{(2)}(t)=\frac{1}{A}\left(\operatorname{cs.sign}\left(x_{1}^{(1)}(t)-x_{1}^{(2)}(t)\right) \sqrt{2 g\left|x_{1}^{(1)}(t)-x_{1}^{(2)}(t)\right|}\right. \\
& \left.-\operatorname{cs.sign}\left(x_{1}^{(2)}(t)-x_{1}^{(3)}(t)\right) \cdot \sqrt{2 g\left|x_{1}^{(2)}(t)-x_{1}^{(3)}(t)\right|}\right)+\eta_{2}(t) \\
& \dot{x}_{1}^{(3)}(t)=\frac{1}{A}\left(\operatorname{cs} \cdot \operatorname{sign}\left(x_{1}^{(2)}(t)-x_{1}^{(3)}(t)\right) \sqrt{2 g\left|x_{1}^{(2)}(t)-x_{1}^{(3)}(t)\right|}\right. \\
& \left.-\operatorname{cs.sign}\left(x_{1}^{(3)}(t)-x_{2}^{(2)}(t)\right) \cdot \sqrt{2 g\left|x_{1}^{(3)}(t)-x_{2}^{(2)}(t)\right|}\right)+\eta_{3}(t) \\
& \dot{x}_{2}^{(2)}(t)=\frac{1}{A}\left(\operatorname{cs} \cdot \operatorname{sign}\left(x_{2}^{(1)}(t)-x_{2}^{(2)}(t)\right) \sqrt{2 g\left|x_{2}^{(1)}(t)-x_{2}^{(2)}(t)\right|}\right. \\
& \left.-\operatorname{cs.sign}\left(x_{2}^{(2)}(t)-x_{2}^{(3)}(t)\right) \cdot \sqrt{2 g\left|x_{2}^{(2)}(t)-x_{2}^{(3)}(t)\right|}\right)+\eta_{4}(t) \\
& \dot{x}_{2}^{(3)}(t)=\frac{1}{A}\left(u_{2}+c \operatorname{ss} \operatorname{sign}\left(x_{2}^{(2)}(t)-x_{2}^{(3)}(t)\right)\right. \\
& \left.\cdot \sqrt{2 g\left|x_{2}^{(2)}(t)-x_{2}^{(3)}(t)\right|}-c s \cdot \sqrt{2 g x_{2}^{(3)}(t)}\right)+\eta_{5}(t)
\end{aligned}
$$


where $x_{1}(k)=\left[x_{1}^{(1)}(t), x_{1}^{(2)}(t), x_{1}^{(3)}(t)\right]^{T} \quad$ is the first subsystem state vector, $x_{2}(t)=\left[x_{2}^{(1)}(t), x_{2}^{(2)}(t), x_{2}^{(3)}(t)\right]^{T}$ is the second subsystem state vector, $A=0.0154 \mathrm{~m}^{2}$ is the cross section of the tanks, $s=5 \times 10^{-5} \mathrm{~m}^{2}$ is the cross section of the connecting pipes, $c=1$ is the outflow coefficient, and $g=9.8 \mathrm{~m} / \mathrm{s}^{2}$ is the standard gravity.

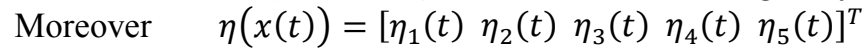
represents the modeling uncertainty and is defined by

$\eta=2 \cdot\left[10^{-2} \sin (0.7 t) 10^{-2} \cos (0.8 t) 10^{-2} \cos (0.5 t)\right.$ $\left.10^{-2} \sin (0.6 t) \quad 10^{-2} \cos (0.7 t)\right]^{T}$

We assume that multiple faults in terms of leakage in tanks 1 and 2 (Local to subsystem 1 ) occur at time $\mathrm{t}_{0}=20$ seconds. Incipient leakage fault dynamics of Subsystem-1 is given by:

$$
\beta\left(t-t_{0}\right) h_{1}\left(x_{1}(t), u_{1}(t)\right)=\left[\begin{array}{c}
\left(1-e^{-0.1\left(t-t_{0}\right)}\right)\left(\frac{\operatorname{sgn}\left[t-t_{0}\right]+1}{2}\right) \sqrt{2 g x_{1}^{(1)}(t)} \\
\left(1-e^{-0.1\left(t-t_{0}\right)}\right)\left(\frac{\operatorname{sgn}\left[t-t_{0}\right]+1}{2}\right) \sqrt{2 g x_{1}^{(2)}(t)} \\
0
\end{array}\right]
$$

The subsystem of our interest in the simulations is subsystem 1. The interconnection function for this subsystem is $g_{1}=-\frac{1}{A} \operatorname{cs} \cdot \operatorname{sign}\left(x_{1}^{(3)}(t)-x_{2}^{(2)}(t)\right) \sqrt{2 g\left|x_{1}^{(3)}(t)-x_{2}^{(2)}(t)\right|}$. Online approximator $\hat{h}_{1}$ is made up of 3-input 3-output sigmoid neural network which consists of 8 hidden layer neurons. The estimator and adaptive law parameters are taken as $\alpha_{i}=2, \gamma_{i}=0.5$, and $\lambda=2$. Using the bounds on the uncertainty and interconnection term, constraint for threshold is calculated as $\rho>\frac{\eta_{M}}{1-\lambda}=0.35$. Therefore, detection threshold is selected as $\rho=0.7$. With this threshold selection, prior to fault, norm of the LFD-1 estimator remains within the threshold as shown in the Fig. 4. However, after the fault has occurred at the $20^{\text {th }}$ second, residual increases above the threshold at $24.18^{\text {th }}$ second.

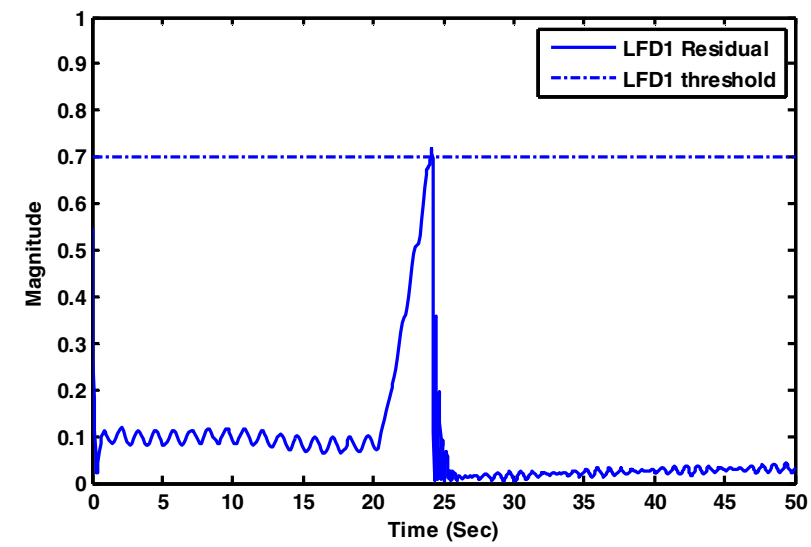

Fig. 4: Residual and threshold of LFD1 when faults occur at tank 1 and 2.

Fig. 5, on the other hand shows the residual generated in LFD2, which reaches the detection threshold at $25.12^{\text {th }}$ second. Since the LFD in subsystem1 detects the fault first, the location of fault is determined to be in subsystem 1 . The residual is finally driven below the threshold in both cases by the tuning law of the OLA. Fig. 6 depicts the actual and estimated local states, when the faults occur within Tanks 1 and 2, i.e. fault is local to subystem 1 . It is clearly seen that the LFD in subsystem 1 is able to approximate the local fault dynamics.

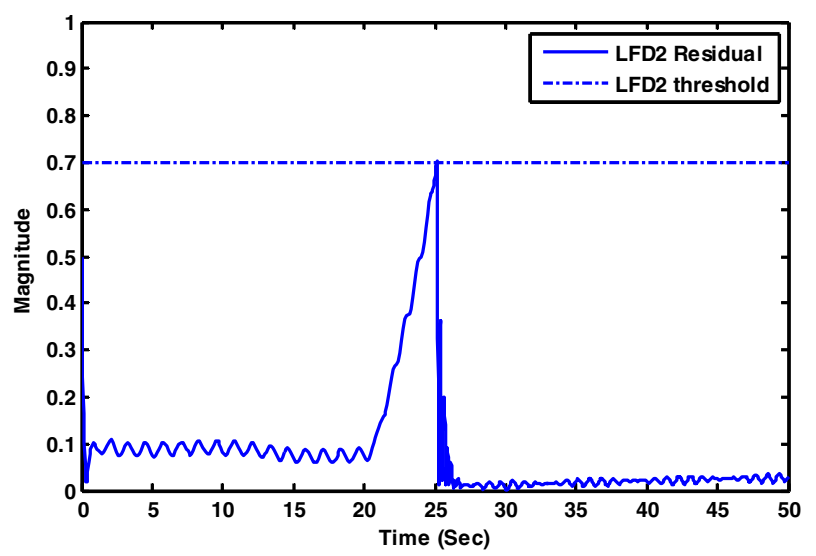

Fig. 5: Residual and threshold of LFD2 when faults occur at tank 1 and 2.

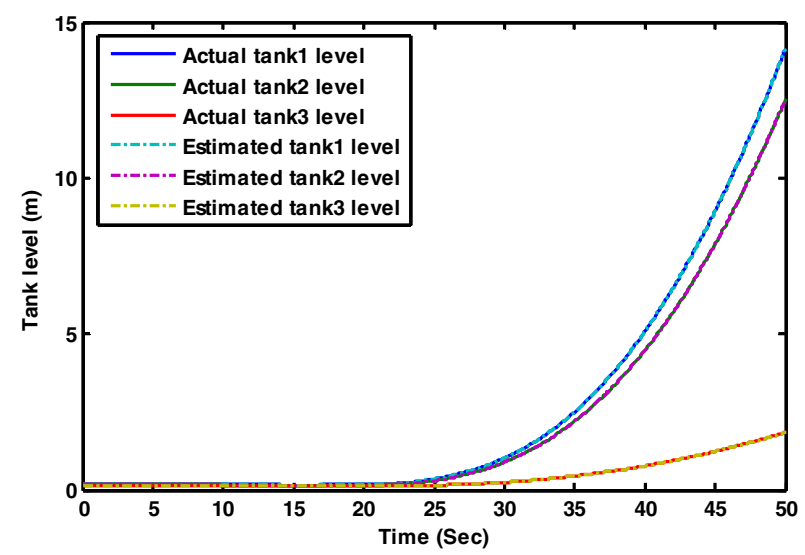

Fig. 6: Estimated and actual states of subsystem1

Fig. 7 depicts the time to failure estimation for local faults in subsystem 1. The initial estimate of TTF is not accurate due to the random selection of weights in the parameter update law. The overall TTF at each point of time is the minimum between the two TTF values at that time.

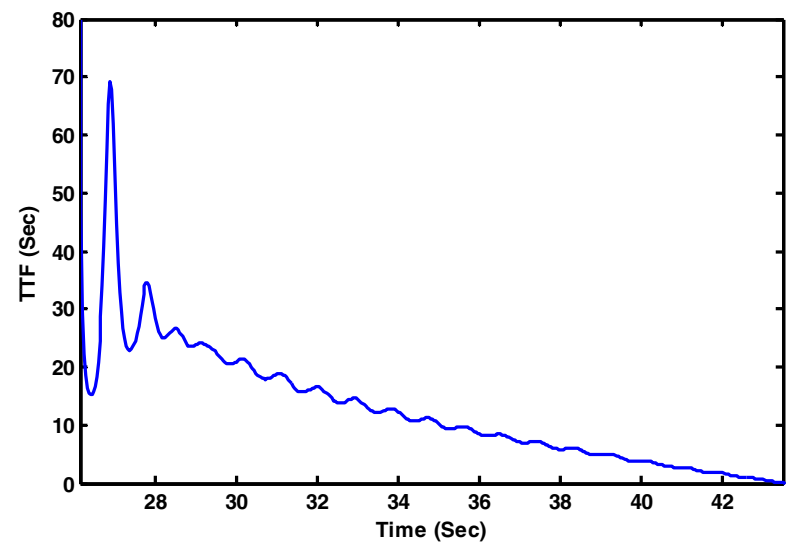

Fig. 7: TTF vs. time for subsystem 1 when the faults are local to subsystem 1. 
A distributed fault diagnosis scheme with overlapping decomposition has been applied on the same simulation problem in [3]. The simulation results in [3] show that although the fault can be detected without a consensus based mechanism, the estimation of fault dynamics will not be accurate. In contrast, simulation results in this paper confirm that when our proposed decentralized scheme is utilized, local and non-local faults can be detected, and more importantly fault dynamics can be estimated almost accurately without using a consensus based mechanism. That means the objective which is detecting and estimating the fault, is reached with a purely decentralized scheme that only uses local states in each LFD. Therefore the LFD network requires less data transmission and fewer neurons. Moreover, the proposed parameter update law and accurate estimation of fault allows estimation of time to failure, which does not exist in [3].

\section{CONCLUSIONS}

The proposed decentralized fault diagnosis and prognosis scheme can be applied on complex industrial systems which are spatially distributed. This model-based scheme does not need interconnection functions to be known and each LFD for the subsystems requires only local states. Therefore less data transmission is needed and each LFD has less data to process which means less computational resources are required. The location determination can help reduce maintenance and repairing costs. Time-to-failure determination was also performed, which will definitely increase the system availability. The only disadvantage of the proposed scheme is that it requires upper bounds on modeling uncertainties and interconnection functions to be known in healthy operating conditions to construct detection thresholds.

\section{REFERENCES}

[1] S. Dash and V. Venkatasubramanian, "Challenges in the industrial applications of fault diagnostic systems," Comput. Chem. Eng., vol. 24, no. 2-7, pp. 785-791, Jul. 2000

[2] R. Isermann, "Model-based fault-detection and diagnosis-status and applications", Annual Reviews in Control, vol. 29, pp. 71-85, 2005.

[3] R. M. G. Ferrari, T. Parisini, and M. M. Polycarpou, "Distributed fault diagnosis with overlapping decompositions: An adaptive approximation approach", IEEE Trans on Automatic Control, vol. 54, no. 4, pp. 794-799, 2009.

[4] M. A. Demetriou and M. M. Polycarpou, "Incipient fault diagnosis of dynamical systems using online approximators," IEEE Trans. on Automatic Control, vol. 43, no. 11, pp. 1612-1617, 1998.

[5] M. M. Polycarpou and A. J. Helmicki, "Automated fault detection and accommodation: A learning systems approach," IEEE Trans. Syst. Man Cybern., vol. 25, no. 11, pp. 1447-1458, Nov. 1995

[6] A. Alessandri and T. Parisini, "Model-based fault diagnosis using nonlinear estimators: a neural approach," in Proc. Amer. Control Conf., June 1997, pp. 903-907.

[7] A. Alessandri, "Fault diagnosis for nonlinear systems using a bank of neural estimators," Comp. in Industry, vol. 52, no.3, pp. 271-289, 2003.

[8] D. Blake and M. Brown, "Simultaneous, multiplicative actuator and sensor fault estimation," Fuzzy Systems Conference 2007, FUZZ-IEEE, pp. 1-6, 2007.

[9] C. J. Lopez-Toribio and R. J. Patton, "Fuzzy observers for nonlinear dynamic systems fault diagnosis," Proc. IEEE Conf. Decision Control, 1998, pp. 84-89.
[10] N. SandUell, P. Varaiya, M. Athans, and M. Safonov, "Survey of decentralized control methods for large scale systems," IEEE Trans. Automat. Control, vol. AC-23, no. 2, pp. 108-128, Apr. 1978.

[11] S. N. Huang, K. K. Tan, and T. H. Lee, "Decentralized control of a class of large-scale nonlinear systems using neural networks," Automatica, vol. 41, pp. 1645-1649, 2005.

[12] S. N. Huang, K. K. Tan, and T. H. Lee, "Nonlinear adaptive control of interconnected systems using neural networks", IEEE Trans on Neural Networks, vol. 17,no. 1, pp. 243-246, 2006.

[13] X. Zhang, "Decentralized fault detection for a class of large-scale nonlinear uncertain systems," Proc. Amer. Control Conf., 2010, pp. $5650-5655$.

[14] T. Parisini, M. M. Polycarpou, and X. Zhang, "Decentralized fault detection in a class of large-scale nonlinear uncertain systems," Proc. IEEE Conf. Decision Control, 2009, pp. 6988-6993.

[15] S. Stankovic, N. Ilic, Z. Djurovic, M. Stankovic, K.H. Johansson, "Consensus based overlapping decentralized fault detection and isolation," Proc. 2010 Conference on Control and Fault-Tolerant Systems (SysTol'10), 2010, pp. 570-575.

[16] B. T. Thumati and S. Jagannathan, "A model based fault detection and prediction scheme for nonlinear multivariable discrete-time systems with asymptotic stability guarantees," IEEE Transactions on Neural Network, vol.21, no. 3, pp.404-423, 2010.

[17] J. Zhang and A. J. Morris, "On-line process fault diagnosis using fuzzy neural networks," Intelligent Systems Engineering, pp. 37-47, 1994.

[18] S. Jagannathan, Neural Network Control of Nonlinear Discrete -time Systems, CRC publications, NY, 2006. 